\title{
SDO/AIA response to coronal hole, quiet Sun, active region, and flare plasma
}

\author{
B. O'Dwyer ${ }^{1}$, G. Del Zanna ${ }^{1}$, H. E. Mason ${ }^{1}$, M. A. Weber ${ }^{2}$, and D. Tripathi ${ }^{1}$ \\ 1 Department of Applied Mathematics and Theoretical Physics, Wilberforce Road, Cambridge, CB3 0WA, UK \\ e-mail: [B.0-Dwyer;G.Del-Zanna;H.E.Mason;D.Tripathi]@damtp.cam.ac.uk \\ 2 Harvard-Smithsonian Center for Astrophysics, 60 Garden St., MS 58, Cambridge, MA 02138, USA \\ e-mail: mweber@cfa.harvard.edu
}

Received 27 April 2010 / Accepted 14 June 2010

\begin{abstract}
Aims. We examine the contribution of spectral lines and continuum emission to the EUV channels of the Atmospheric Imaging Assembly (AIA) on the Solar Dynamics Observatory (SDO) in different regions of the solar atmosphere.

Methods. Synthetic spectra were obtained using the CHIANTI atomic database and sample differential emission measures for coronal hole, quiet Sun, active region and flare plasma. These synthetic spectra were convolved with the effective area of each channel, in order to determine the dominant contribution in different regions of the solar atmosphere.

Results. We highlight the contribution of particular spectral lines which under certain conditions can affect the interpretation of $\mathrm{SDO} / \mathrm{AIA}$ data.
\end{abstract}

Key words. Sun: activity - Sun: atmosphere - Sun: corona - Sun: UV radiation

\section{Introduction}

The Atmospheric Imaging Assembly (AIA; Title et al. 2006) on the Solar Dynamics Observatory (SDO; launched 11 February, 2010; Schwer et al. 2002) is a set of normal-incidence imaging telescopes designed to acquire images of the solar atmosphere in a variety of extreme ultraviolet (EUV), ultraviolet and visiblelight wavelength bands. The instrument observes solar plasma from photospheric to coronal temperatures, taking full-disk images, with high spatial resolution ( $\sim 0.6$ arcsec pixels $)$ and with a cadence of $10 \mathrm{~s}$ or better.

Of the seven EUV channels six are expected ${ }^{1}$ to be dominated by iron emission lines: $94 \AA$ (Fe XVIII), $131 \AA$ (Fe VIII, Fe XX, Fe XXIII), $171 \AA$ (Fe IX), $193 \AA$ (Fe XII, Fe XXIV), $211 \AA$ (Fe XIV), $335 \AA$ (Fe XVI), with the remaining channel $304 \AA$ dominated by He II. In this letter we examine the contribution of spectral lines and continuum emission to each of the EUV channels in order to determine what the dominant contribution to each channel is in different regions of the solar atmosphere. It is timely for us to do so now in order to ensure the correct interpretation of observed features in SDO/AIA data. Analysis of this kind has been performed previously for the Transition Region and Coronal Explorer (TRACE; Handy et al. 1999) $173 \AA$ and $195 \AA$ passbands and the Extreme-ultraviolet Imaging Telescope (EIT; Delaboudinière et al. 1995) $171 \AA$, $195 \AA$ and $284 \AA$ passbands (cf., Del Zanna \& Mason 2003; Del Zanna et al. 2003; Phillips et al. 2005; Tripathi et al. 2006).

\footnotetext{
1 Atmospheric Imaging Assembly Concept Study Report, Appendix A (Science Plan), http://aia. lmsal. com/public/CSR. htm
}

\section{Analysis}

Under the assumption that a plasma is optically thin and in ionization equilibrium the observed intensity of a spectral emission line can be expressed as

$I_{\mathrm{ob}}=A(\mathrm{z}) \int_{T_{\mathrm{e}}} G\left(T_{\mathrm{e}}, N_{\mathrm{e}}\right) \varphi\left(T_{\mathrm{e}}\right) \mathrm{d} T_{\mathrm{e}}$

where $A(\mathrm{z})$ is the elemental abundance, $T_{\mathrm{e}}$ is the electron temperature, and $N_{\mathrm{e}}$ is the electron number density. The contribution function, $G\left(T_{\mathrm{e}}, N_{\mathrm{e}}\right)$, contains the relevant atomic parameters for each line and can be obtained using equilibrium ionization balance calculations. For this purpose we have used CHIANTI v. 6.0.1 (Dere et al. 1997, 2009) atomic data and ionization equilibrium. The quantity $\varphi\left(T_{\mathrm{e}}\right)$ is known as the differential emission measure (DEM) which is defined as

$\varphi\left(T_{\mathrm{e}}\right)=N_{\mathrm{e}}^{2} \frac{\mathrm{d} h}{\mathrm{~d} T_{\mathrm{e}}}$

where $\mathrm{h}$ is the line-of-sight coordinate.

Included in Fig. 1 are sample DEMs for coronal hole $(\mathrm{CH})$, quiet Sun (QS), active region (AR) and flare plasma. The $\mathrm{CH}$ DEM (Del Zanna 1999) and QS DEM (Andretta et al. 2003) were obtained from measurements by the Coronal Diagnostic Spectrometer (CDS; Harrison et al. 1995) on the Solar and Heliospheric Observatory. The AR DEM (O'Dwyer et al., in prep.) was obtained from AR intensities measured by the EUV Imaging Spectrometer (EIS; Culhane et al. 2007) on Hinode. The flare DEM was based on observations of an M2 X-ray class flare by Dere \& Cook (1979).

Theoretical spectra have been calculated with these DEMs, using CHIANTI v. 6.0.1. The photospheric abundances of 


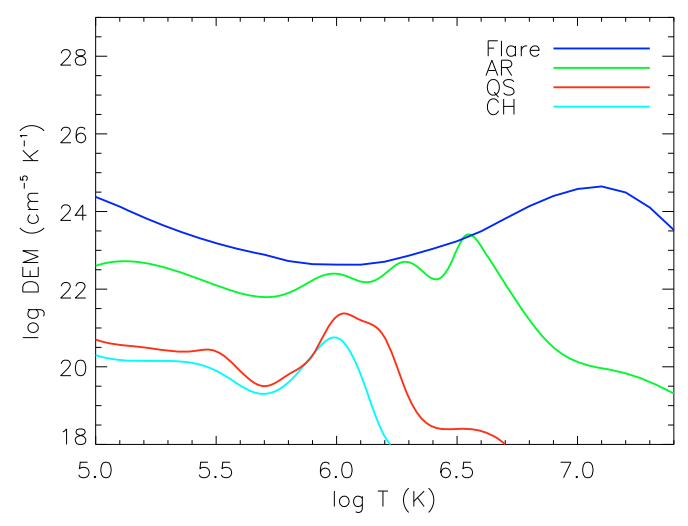

Fig. 1. Plot of DEM curves for coronal hole, quiet Sun, active region and flare plasma (see text for references).

Asplund et al. (2009) were used in calculating the $\mathrm{CH}$, QS and AR spectra. Density values of $N_{\mathrm{e}}=2 \times 10^{8} \mathrm{~cm}^{-3}$, $N_{\mathrm{e}}=5 \times 10^{8} \mathrm{~cm}^{-3}$ and $N_{\mathrm{e}}=5 \times 10^{9} \mathrm{~cm}^{-3}$ were used in calculating the $\mathrm{CH}$, QS and AR spectra respectively. These are the same density values as those used to calculate the contribution functions for the lines which were used to constrain the DEM curves for the $\mathrm{CH}, \mathrm{QS}$ and $\mathrm{AR}$ cases. For the flare spectrum a value of $N_{\mathrm{e}}=1 \times 10^{11} \mathrm{~cm}^{-3}$ and the solar coronal abundances of Feldman et al. (1992) were used. The use of either photospheric or coronal abundances in calculating the synthetic spectra reflects the original use of either photospheric or coronal abundances in generating the DEM curves. These synthetic spectra were then convolved with the effective area of each channel. The effective areas were obtained from P. Boerner (2009, priv. comm.), with the exception of the $171 \AA$ and $335 \AA$ channels for which updated versions were used obtained from Solarsoft (12 July, 2010).

\section{Results}

Table 1 lists those spectral lines which contribute more than 3\% to the total emission in each channel for $\mathrm{CH}, \mathrm{QS}, \mathrm{AR}$ and flare plasma. Also included is the fractional contribution of the continuum emission for any case where the continuum contributes more than $3 \%$ to the total emission in a channel. Synthetic spectra for each of the channels are displayed in Figs. 2-8. For every channel each spectrum has been divided by the peak intensity of the strongest spectrum. Weaker spectra have been scaled by factors indicated in each figure.

The $94 \AA$ channel is expected ${ }^{1}$ to observe the Fe XVIII $93.93 \AA$ line $[\log T[\mathrm{~K}] \sim 6.85]$ in flaring regions. For both AR and flare plasma (see Fig. 2) the dominant contribution comes from the Fe XVIII $93.93 \AA$ line. However, for the $\mathrm{CH}$ and QS spectrum (see Fig. 2) the dominant contribution comes from the Fe X $94.01 \AA \operatorname{line}[\log T \sim 6.05]$.

In flaring regions the $131 \AA$ channel is expected ${ }^{1}$ to observe the Fe XX $132.84 \AA$ and Fe XXIII $132.91 \AA$ lines. However, for the flare spectrum (see Fig. 3 ) the dominant contribution comes from the Fe XXI $128.75 \AA$ line [ $\log T \sim 7.05]$. The combined contribution of the Fe XX $132.84 \AA$ and Fe XXIII 132.91 $\AA$ lines is less than ten percent of the total emission. For $\mathrm{CH}$ observations the $131 \AA$ channel is expected ${ }^{1}$ to be dominated by Fe VIII lines $[\log T \sim 5$.6]. From our simulations, the dominant contribution for $\mathrm{CH}$ and QS plasma (see Fig. 3) does come from Fe VIII lines,
Table 1. Predicted AIA count rates.

\begin{tabular}{|c|c|c|c|c|c|c|c|}
\hline & \multirow[t]{2}{*}{ Ion } & \multirow{2}{*}{$\begin{array}{l}\lambda \\
\AA\end{array}$} & \multirow{2}{*}{$\begin{array}{c}T_{\mathrm{p}}{ }^{a} \\
\mathrm{~K}\end{array}$} & \multicolumn{4}{|c|}{ Fraction of total emission } \\
\hline & & & & $\mathrm{CH}$ & QS & AR & FL \\
\hline \multirow[t]{7}{*}{$94 \AA$} & Mg VIII & 94.07 & 5.9 & 0.03 & - & - & - \\
\hline & Fe XX & 93.78 & 7.0 & - & - & - & 0.10 \\
\hline & Fe XVIII & 93.93 & 6.85 & - & - & 0.74 & 0.85 \\
\hline & $\mathrm{Fe} \mathrm{X}$ & 94.01 & 6.05 & 0.63 & 0.72 & 0.05 & - \\
\hline & Fe VIII & 93.47 & 5.6 & 0.04 & - & - & - \\
\hline & Fe VIII & 93.62 & 5.6 & 0.05 & - & - & - \\
\hline & Cont. & & & 0.11 & 0.12 & 0.17 & - \\
\hline \multirow[t]{6}{*}{$131 \AA$} & OVI & 129.87 & 5.45 & 0.04 & 0.05 & - & - \\
\hline & Fe XXIII & 132.91 & 7.15 & - & - & - & 0.07 \\
\hline & Fe XXI & 128.75 & 7.05 & - & - & - & 0.83 \\
\hline & Fe VIII & 130.94 & 5.6 & 0.30 & 0.25 & 0.09 & - \\
\hline & Fe VIII & 131.24 & 5.6 & 0.39 & 0.33 & 0.13 & - \\
\hline & Cont. & & & 0.11 & 0.20 & 0.54 & 0.04 \\
\hline \multirow[t]{4}{*}{$171 \AA$} & Ni XIV & 171.37 & 6.35 & - & - & 0.04 & - \\
\hline & Fe $\mathrm{X}$ & 174.53 & 6.05 & - & 0.03 & - & - \\
\hline & Fe IX & 171.07 & 5.85 & 0.95 & 0.92 & 0.80 & 0.54 \\
\hline & Cont. & & & - & - & - & 0.23 \\
\hline \multirow[t]{14}{*}{$193 \AA$} & $\mathrm{OV}$ & 192.90 & 5.35 & 0.03 & - & - & - \\
\hline & Ca XVII & 192.85 & 6.75 & - & - & - & 0.08 \\
\hline & Ca XIV & 193.87 & 6.55 & - & - & 0.04 & - \\
\hline & Fe XXIV & 192.03 & 7.25 & - & - & - & 0.81 \\
\hline & Fe XII & 195.12 & 6.2 & 0.08 & 0.18 & 0.17 & - \\
\hline & Fe XII & 193.51 & 6.2 & 0.09 & 0.19 & 0.17 & - \\
\hline & Fe XII & 192.39 & 6.2 & 0.04 & 0.09 & 0.08 & - \\
\hline & Fe XI & 188.23 & 6.15 & 0.09 & 0.10 & 0.04 & - \\
\hline & Fe XI & 192.83 & 6.15 & 0.05 & 0.06 & - & - \\
\hline & Fe XI & 188.30 & 6.15 & 0.04 & 0.04 & - & - \\
\hline & Fex & 190.04 & 6.05 & 0.06 & 0.04 & - & - \\
\hline & Fe IX & 189.94 & 5.85 & 0.06 & - & - & - \\
\hline & Fe IX & 188.50 & 5.85 & 0.07 & - & - & - \\
\hline & Cont. & & & - & - & 0.05 & 0.04 \\
\hline \multirow[t]{11}{*}{$211 \AA$} & CrIX & 210.61 & 5.95 & 0.07 & - & - & - \\
\hline & CaXVI & 208.60 & 6.7 & - & - & - & 0.09 \\
\hline & Fe XVII & 204.67 & 6.6 & - & - & - & 0.07 \\
\hline & Fe XIV & 211.32 & 6.3 & - & 0.13 & 0.39 & 0.12 \\
\hline & Fe XIII & 202.04 & 6.25 & - & 0.05 & - & - \\
\hline & Fe XIII & 203.83 & 6.25 & - & - & 0.07 & - \\
\hline & Fe XIII & 209.62 & 6.25 & - & 0.05 & 0.05 & - \\
\hline & Fe XI & 209.78 & 6.15 & 0.11 & 0.12 & - & - \\
\hline & Fe $\mathrm{X}$ & 207.45 & 6.05 & 0.05 & 0.03 & - & - \\
\hline & Ni XI & 207.92 & 6.1 & 0.03 & - & - & - \\
\hline & Cont. & & & 0.08 & 0.04 & 0.07 & 0.41 \\
\hline \multirow[t]{5}{*}{$304 \AA$} & He II & 303.786 & 4.7 & 0.33 & 0.32 & 0.27 & 0.29 \\
\hline & He II & 303.781 & 4.7 & 0.66 & 0.65 & 0.54 & 0.58 \\
\hline & Ca XVIII & 302.19 & 6.85 & - & - & - & 0.05 \\
\hline & Si XI & 303.33 & 6.2 & - & - & 0.11 & - \\
\hline & Cont. & & & - & - & - & - \\
\hline \multirow[t]{9}{*}{$335 \AA$} & $\mathrm{Al} x$ & 332.79 & 6.1 & 0.05 & 0.11 & - & - \\
\hline & Mg VIII & 335.23 & 5.9 & 0.11 & 0.06 & - & - \\
\hline & Mg VIII & 338.98 & 5.9 & 0.11 & 0.06 & - & - \\
\hline & Si IX & 341.95 & 6.05 & 0.03 & 0.03 & - & - \\
\hline & Si VIII & 319.84 & 5.95 & 0.04 & - & - & - \\
\hline & Fe XVI & 335.41 & 6.45 & - & - & 0.86 & 0.81 \\
\hline & Fe XIV & 334.18 & 6.3 & - & 0.04 & 0.04 & - \\
\hline & Fe X & 184.54 & 6.05 & 0.13 & 0.15 & - & - \\
\hline & Cont. & & & 0.08 & 0.05 & - & 0.06 \\
\hline
\end{tabular}

Notes. The count rates are normalised for each channel. Coronal hole $(\mathrm{CH})$, quiet Sun (QS), active region (AR) and flare (FL) plasma. ${ }^{(a)} T_{\mathrm{p}}$ corresponds to the log of the temperature of maximum abundance. 


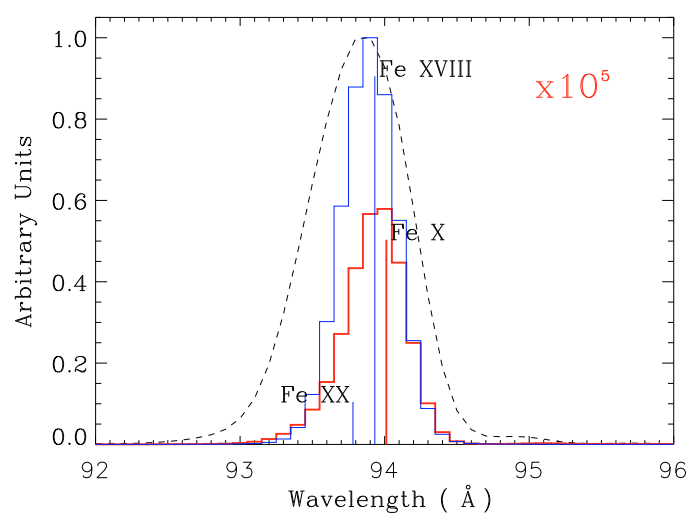

Fig. 2. Flare (blue) and QS (red) synthetic spectra for the $94 \AA$ channel. The effective area is overplotted as a dashed line. The peak intensities of stronger lines are indicated. The weaker spectrum has been scaled by a factor indicated in the figure.

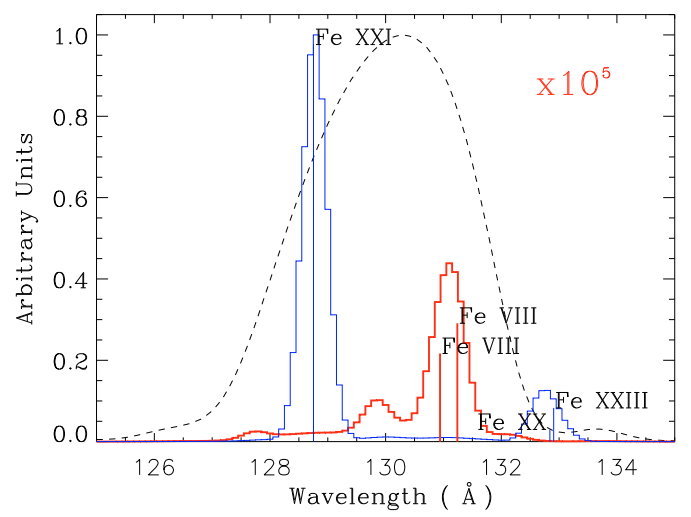

Fig. 3. Same as Fig. 2 for the $131 \AA$ A channel.



Fig. 4. Same as Fig. 2 for the $171 \AA$ A channel.

but with a significant contribution from continuum emission. For the AR spectrum the dominant contribution comes from continuum emission, with a significant contribution from Fe VIII lines.

The $171 \AA$ channel is expected ${ }^{1}$ to observe the Fe IX $171.07 \AA$ line $[\log T \sim 5.85]$ for QS observations. For CH, QS (see Fig. 4) and AR plasma the dominant contribution comes from the Fe IX 171.07 $\AA$ line. For the flare spectrum (see Fig. 4) the dominant contribution comes from the

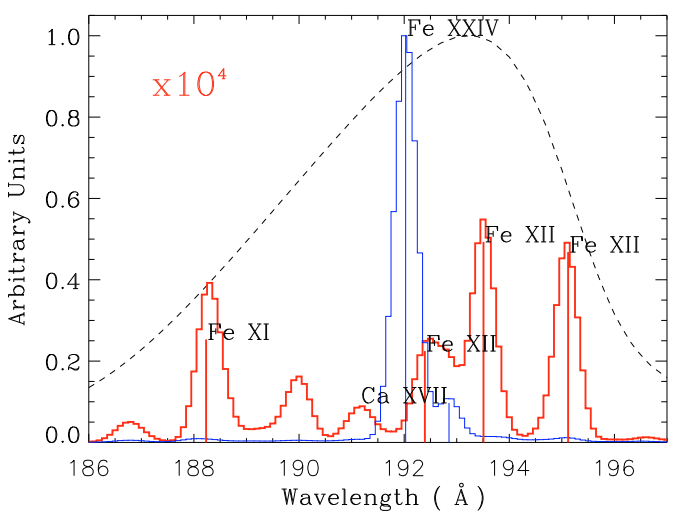

Fig. 5. Same as Fig. 2 for the $193 \AA$ channel.

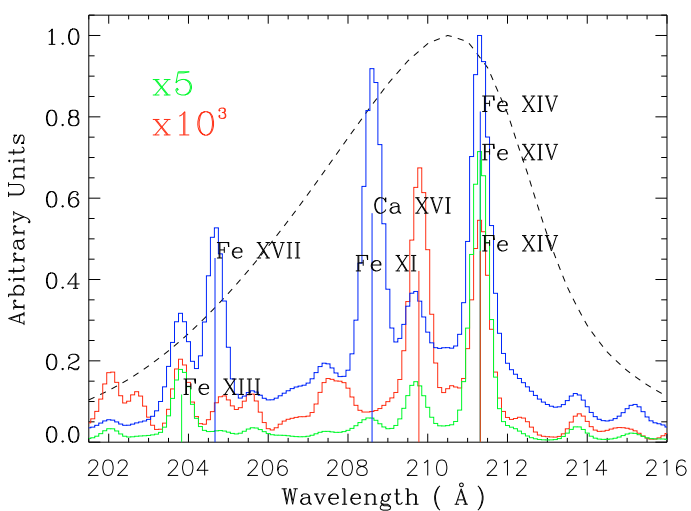

Fig. 6. Same as Fig. 2 for the $211 \AA$ A channel. The AR synthetic spectrum (green) is also included.

Fe IX $171.07 \AA$ line, with a significant contribution from continuum emission. The $171 \AA$ channel has a response to flarelike temperatures due to contributions from Fe XX lines. Phillips et al. (2005) highlighted the response of the TRACE $173 \AA$ passband at flarelike temperatures, including the contribution of continuum emission to the passband at high temperatures. Raftery et al. (in prep.) have examined the response of the AIA $171 \AA$ channel, highlighting the high temperature response of the channel for flare plasma.

The $193 \AA$ channel is expected ${ }^{1}$ to be dominated by Fe XII lines $[\log T \sim 6.2]$ for $\mathrm{QS}$ and AR observations. For the QS (see Fig. 5) and AR spectra the dominant contribution does come from Fe XII lines, but with a significant contribution from Fe XI lines. In flaring regions the $193 \AA$ channel is expected ${ }^{1}$ to be dominated by the Fe XXIV $192.03 \AA$ line $[\log T \sim 7.25]$, which is indeed the case (see Fig. 5). However, for the $\mathrm{CH}$ spectrum there are significant contributions from Fe IX, Fe XI and Fe XII lines.

The $211 \AA$ channel is expected ${ }^{1}$ to observe the Fe XIV $211.32 \AA$ line $[\log T \sim 6.3]$ for AR observations. For AR plasma (see Fig. 6) the dominant contribution comes from the Fe XIV 211.32 $\AA$ line, as well as a significant contribution from Fe XIII lines. The fractional contribution of Fe XIII lines to the total emission is 0.24 , while the contribution of Fe XIV lines is 0.41 . For the $\mathrm{CH}$ spectrum the most significant contributions come from Fe XI, Fe X, Fe IX and CrIX lines, as well as continuum emission. For the QS spectrum (see Fig. 6) 
the most significant contributions come from Fe XI, Fe XIII and Fe XIV. For the flare spectrum (see Fig. 6) the most significant contribution comes from continuum emission. The $211 \AA$ channel has a response at high temperatures due to contributions from Fe XVII and Ca XVI lines.

As expected ${ }^{1}$ the $304 \AA$ channel is dominated by the two He II $303.8 \AA \operatorname{lines}[\log T \sim 4$ 4] for $\mathrm{CH}, \mathrm{QS}, \mathrm{AR}$ and flare plasma (see Fig. 7). However, in QS off-limb the dominant contribution to this channel would be expected to come from the Si XI $303.33 \AA$ line $[\log T \sim 6.2]$. This line is formed at a much higher temperature than the chromospheric He II $303.8 \AA$ lines which dominate on-disk. The contribution of Si XI $303.33 \AA$ to the He II $303.8 \AA$ lines has been highlighted previously (Tousey et al. 1965). The $304 \AA$ channel has a small response to flarelike temperatures due to a contribution from the Ca XVIII $302.19 \AA$ line. Due to the anomalous behaviour of He II (see Andretta et al. 2003, and references therein) the predicted line intensities are under-estimated by large factors. For this reason correction factors based on observations have been applied. For the QS case a factor of 24.0 was used based on the observed intensity of the He II $304 \AA$ line from Andretta et al. (2003). For the $\mathrm{CH}$ and AR cases correction factors of 19.3 and 4.82 have been adopted respectively. These values are based on the observed intensity of the He II $304 \AA$ line from Vernazza \& Reeves (1978). The correction factor adopted for the AR case was also used for the flare case.

The $335 \AA$ channel is expected ${ }^{1}$ to observe the Fe XVI $335.41 \AA$ line $[\log T \sim 6.45]$ for AR observations. For AR (see Fig. 8) and flare plasma the dominant contribution does indeed come from the Fe XVI $335.41 \AA$ line. However, for the $\mathrm{CH}$ spectrum the most significant contributions come from Mg VIII and FeX lines and for QS plasma (see Fig. 8) the most significant contributions come from $\mathrm{Mg}$ VIII, $\mathrm{Al} \mathrm{X}$ and Fe $\mathrm{X}$ lines.

Changing the density values used in creating the synthetic spectra would affect the absolute intensities of the density sensitive lines, as well as the continuum. However for those cases considered here we note that varying the density by e.g. a factor of two only changes the fractional contribution of a particular line to a channel by at most four percent.

The AR DEM curve we have used to create the synthetic spectra was constrained using lines from low first ionization potential (FIP) elements, such as iron. For any synthetic spectrum created from a DEM curve of this kind the absolute intensities of lines from low-FIP elements are independent of the choice of elemental abundances, provided that the same abundances are used in creating the synthetic spectrum, as were used in generating the DEM curve. For lines from high-FIP elements the use of photospheric abundances increases the absolute intensities of the lines by a factor of $\sim 4$ relative to the intensities obtained using coronal abundances. We note that, with the exception of the $304 \AA$ channel, the contribution of lines from high-FIP elements to each channel is very small, of a few percent at most (cf. Table 1). As a result using coronal rather than photospheric abundances will not dramatically affect the fractional contribution of lines to each channel.

\section{Conclusions and discussion}

We have determined what the dominant contribution to each AIA channel is in different regions of the solar atmosphere. In general our findings are in agreement with expectations ${ }^{1}$. However

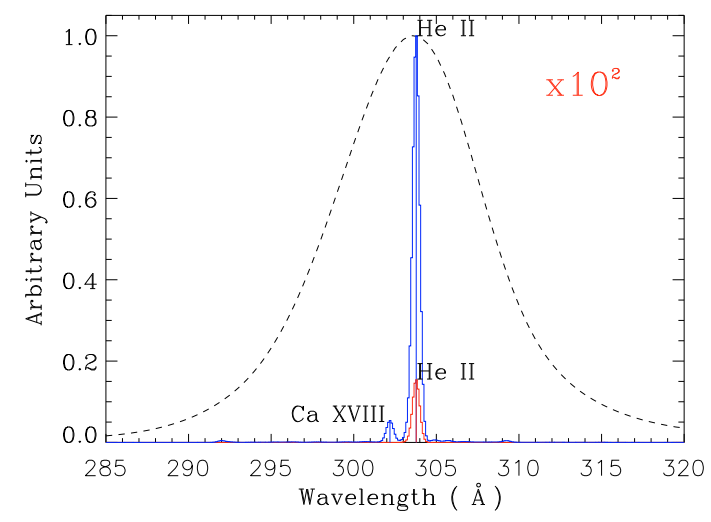

Fig. 7. Same as Fig. 2 for the $304 \AA$ channel. Note the peak intensities of the two He II $303.8 \AA$ lines have been combined.



Fig. 8. Same as Fig. 2 for the $335 \AA$ channel, except that the AR synthetic spectrum (green) is included instead of the flare spectrum.

Table 2. Count rates for each channel for the $\mathrm{CH}, \mathrm{QS}, \mathrm{AR}$ and flare synthetic spectra relative to the count rate for the $94 \AA$ channel for the $\mathrm{CH}$ synthetic spectrum.

\begin{tabular}{ccccc}
\hline \hline Channel & CH & QS & AR & Flare \\
\hline $94 \AA$ & 1.00 & 6.09 & $1.11 \times 10^{3}$ & $9.38 \times 10^{5}$ \\
$131 \AA$ & $1.42 \times 10^{1}$ & $4.73 \times 10^{1}$ & $3.92 \times 10^{3}$ & $6.61 \times 10^{6}$ \\
$171 \AA$ & $1.10 \times 10^{3}$ & $4.08 \times 10^{3}$ & $7.35 \times 10^{4}$ & $4.36 \times 10^{5}$ \\
$193 \AA$ & $1.87 \times 10^{2}$ & $1.84 \times 10^{3}$ & $6.97 \times 10^{4}$ & $8.96 \times 10^{6}$ \\
$211 \AA$ & $3.39 \times 10^{1}$ & $3.30 \times 10^{2}$ & $3.29 \times 10^{4}$ & $6.14 \times 10^{5}$ \\
$304 \AA$ & $2.55 \times 10^{2}$ & $8.21 \times 10^{2}$ & $9.60 \times 10^{3}$ & $5.93 \times 10^{5}$ \\
$335 \AA$ & 3.05 & $1.62 \times 10^{1}$ & $4.82 \times 10^{3}$ & $1.43 \times 10^{5}$ \\
\hline
\end{tabular}

we note that with the $131 \AA$ channel for our flare spectrum the dominant contribution does not come from the Fe XX $132.84 \AA$ or Fe XXIII 132.91 $\AA$ lines, but instead from the Fe XXI 128.75 line.

It is important to note that when using AIA channels to observe regions of the solar atmosphere other than those for which each channel was designed the contribution of particular spectral lines and continuum emission can affect the interpretation of the observed features. We note that the dominant contribution to the $94 \AA$ channel for QS plasma comes from the Fe X $94.01 \AA$ line. We also note the contribution of continuum emission to the $131 \AA$ channel in ARs. In addition we note the significant 
contribution of continuum emission to the $211 \AA$ channel in flaring regions.

The DEMs which we have used are intended to give an indication of which lines contribute to the SDO/AIA EUV channels under different solar conditions. Different DEMs will naturally give different contributions. For example with the QS DEM in CHIANTI for the $335 \AA$ channel there is a significant contribution (32\% of the total emission) from the Fe XVI $335.41 \AA$ line. The QS DEM that we have used falls off more quickly at higher temperatures than the one in CHIANTI, and as a result the predicted contribution of the Fe XVI $335.41 \AA$ line is much lower (2\% of the total emission). Another example is that with the $\mathrm{CH}$ DEM in CHIANTI for the $193 \AA$ channel there is a significant contribution from Fe VIII lines (44\% of the total emission). With the $\mathrm{CH}$ DEM that we have used the predicted contribution of Fe VIII lines is lower (9\% of the total emission). The CH DEM in CHIANTI peaks at a lower temperature than the one we have used, and as a result the fractional contribution of lower temperature lines is greater. Del Zanna et al. (2003) highlighted the contribution of Fe VIII lines to the EIT $195 \AA$ passband during a $\mathrm{CH}$ observation.

No channel is predicted to be strictly isothermal, in the sense that emission from plasma formed at different temperatures contributes to the signal. For this reason extreme care must be taken when using ratios of SDO/AIA channels to derive isothermal temperatures. Detailed inversion (DEM) and forward modelling are required in most cases.
Acknowledgements. B.O.D., G.D.Z., H.E.M., D.T. acknowledge STFC. M.W. was supported under AIA subcontract SP02H1701R from Lockheed-Martin. B.O.D. was supported by funding from the Gates Cambridge Trust. B.O.D. would like to thank the SAO for providing support for a visit during which this work was initiated. The authors thank Dr. Paul Boerner of the AIA Team for calculating the AIA effective areas. CHIANTI is a collaborative project involving researchers at NRL (USA), and the Universities of: Cambridge (UK), George Mason (USA), and Florence (Italy).

\section{References}

Andretta, V., Del Zanna, G., \& Jordan, S. D. 2003, A\&A, 400, 737 Asplund, M., Grevesse, N., Sauval, A. J., \& Scott, P. 2009, ARA\&A, 47, 481 Culhane, J. L., Harra, L. K., James, A. M., et al. 2007, Sol. Phys., 243, 19 Del Zanna, G. 1999, Ph.D. Thesis, Univ. of Central Lancashire

Del Zanna, G., \& Mason, H. E. 2003, A\&A, 406, 1089

Del Zanna, G., Bromage, B. J. I., \& Mason, H. E. 2003, A\&A, 398, 743

Delaboudinière, J., Artzner, G. E., Brunaud, J., et al. 1995, Sol. Phys., 162, 291 Dere, K. P., \& Cook, J. W. 1979, ApJ, 229, 772

Dere, K. P., Landi, E., Mason, H. E., Monsignori Fossi, B. C., \& Young, P. R. 1997, A\&AS, 125, 149

Dere, K. P., Landi, E., Young, P. R., et al. 2009, A\&A, 498, 915

Feldman, U., Mandelbaum, P., Seely, J. F., Doschek, G. A., \& Gursky, H. 1992, ApJS, 81, 387

Handy, B. N., Acton, L. W., Kankelborg, C. C., et al. 1999, Sol. Phys., 187, 229 Harrison, R. A., Sawyer, E. C., Carter, M. K., et al. 1995, Sol. Phys., 162, 233

Phillips, K. J. H., Chifor, C., \& Landi, E. 2005, ApJ, 626, 1110

Schwer, K., Lilly, R. B., Thompson, B. J., \& Brewer, D. A. 2002, AGU Fall Meeting Abstracts, $\mathrm{C} 1$

Tousey, R., Austin, W. E., Purcell, J. D., \& Widing, K. G. 1965, Ann. Astrophys., 28,755

Tripathi, D., Del Zanna, G., Mason, H. E., \& Chifor, C. 2006, A\&A, 460, L53 Vernazza, J. E., \& Reeves, E. M. 1978, ApJS, 37, 485 